\title{
How two share two tasks: evidence of a social psychological refractory period effect
}

\author{
Roman Liepelt • Wolfgang Prinz
}

Received: 18 January 2011 / Accepted: 16 April 2011 / Published online: 5 May 2011

(C) Springer-Verlag 2011

\begin{abstract}
A strong assumption shared by major theoretical approaches to cognition posits that the human cognitive system has a limited capacity for information processing. Evidence supporting this claim comes from the dual-task paradigm in which one cognitive system has to process two tasks simultaneously. In this study, we examined whether bottleneck-like processing can also be elicited when a dual task is shared between two individuals. Under dual-task instructions giving priority to Task 1 , we found evidence of a psychological refractory period effect in dual-task and joint-task conditions. Under equal priority instructions, we replicated the finding of a psychological refractory period effect in the dual-task, but not in the joint-task condition. These findings are in line with the assumption that a social psychological refractory period effect can be induced across two individuals. We suggest that this effect is due to taskspecific monitoring requirements. We discuss our findings with respect to both dual-task and joint action theories.
\end{abstract}

Keywords Joint action $\cdot$ Social PRP · Dual task

\section{Introduction}

Major theoretical approaches to cognition posit that the human cognitive system has a limited amount of capacity

R. Liepelt $\cdot$ W. Prinz

Department of Psychology, Max Planck Institute For Human

Cognitive and Brain Sciences, Leipzig, Germany

\section{R. Liepelt ( $\square)$}

Department of Psychology, Junior Group "Neurocognition of Joint

Action”, Westfälische Wilhelms University,

Fliednerstrasse 21, 48149 Münster, Germany

e-mail: roman.liepelt@uni-muenster.de for information processing and task performance. Major evidence for this claim came from dual-task research, showing that simultaneous performance of two choice reaction time (RT) tasks leads to dramatic slowing of the second task (Pashler 1994; Telford 1931; Welford 1952). Since the 1950s, the psychological refractory period (PRP) paradigm has become one of the most important paradigms to visualize and test such processing limitations. Typical findings using this paradigm are that RTs and/or error rates in the second task (RT 2) increase when the stimulus onset asynchrony (SOA) is reduced (Fig. 1). This RT 2 pattern is denoted as the PRP effect and reflects additional performance costs in dual tasks (Pashler 1994; Telford 1931). RT 1 is typically found to be relatively independent of the SOA manipulation (Pashler and Johnston 1989).

A prominent model developed to explain this effect is the central Response Selection Bottleneck model (Pashler 1984, 1994). According to this model, peripheral stages (perception and motor execution) can be processed with any other stage in parallel, while the central response selection stage can only be processed in one task at a time (Fig. 1). In particular, it is assumed that the response selection stage is postponed in one task until the processing of the response selection stage in the other task is completed. According to this model, dual-task costs have been attributed to a structural capacity limitation, which may be located at the response selection stage (Pashler 1994) when one person has to perform two tasks simultaneously.

However, there is now evidence suggesting that the existence of a Response Selection Bottleneck may be an artifact of task instructions and the respective dual-task conditions given (Schumacher et al. 2001). Meyer and Kieras (1997a, b, 1999) developed an adaptive executive control model derived from the EPIC (executive processing-interactive control) architecture to account for the finding of dual-task 


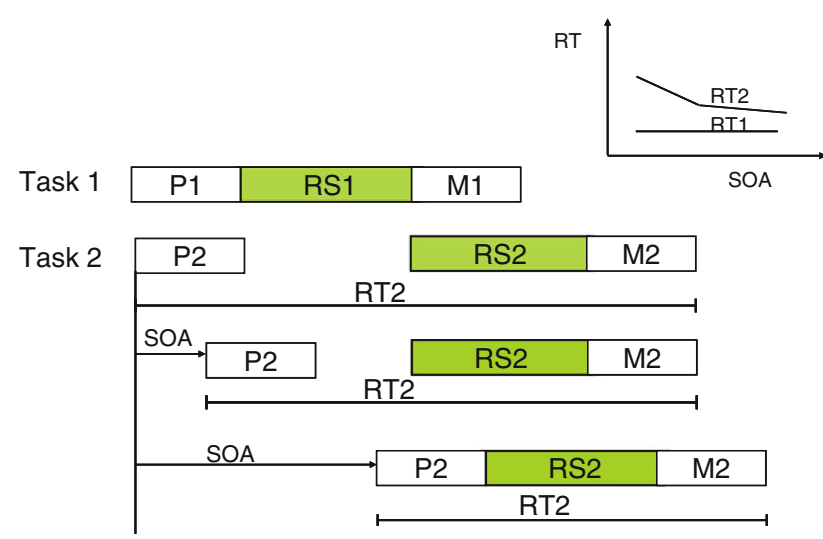

Fig. 1 Bottleneck processing in dual tasks (within one person). Three stages of processing ( $P$ perception, $R S$ response selection, $M$ motor execution) for Task 1 and Task 2 at short and long Stimulus Onset Asynchronies (SOAs). When the SOA is long, the bottleneck stages (Stage RS) for the two tasks are required at non-overlapping times, so both tasks can proceed without interruption. When the SOA is short, the bottleneck stage (Stage RS) for Task 2 must wait for the bottleneck stage in Task 1 to be completed, so Task 2 is slowed. This assumption explains the finding of the typical Reaction Time on Task 2 (RT 2) slowing with decreasing SOA (PRP effect) and the relative independence of Reaction Time on Task 1 (RT 1) from the stimulus onset asynchrony (SOA), as shown in the schematic drawing in the right upper panel

costs under simultaneous dual-task performance. Dual-task costs emerge when strategic bottlenecks are introduced according to specific task requirements (e.g., when required by task instructions, when both tasks overlap in input or output processing, or when participants have only a small amount of practice with the dual-task situation). According to the EPIC model, strategic bottlenecks can theoretically be introduced at any processing stage, for example, perception, central response selection, or motor stages (Meyer and Kieras 1997a, b), when inter-task coordination is required (Liepelt et al. in pressa). Given that some studies provided evidence for dual-task cost elimination (Schumacher et al. 2001) under optimal task conditions and sufficient practice, an account based on optional task-scheduling strategies may provide a more veridical explanation for the PRP effect. Strategic bottlenecks are adopted to optimize task performance and fulfill task demands.

Instead of focusing on individual task performance more recently many researches became also interested in social task performance measuring action observation (Brass et al. 2000; Liepelt et al. 2008) or real-time social interaction (Sebanz et al. 2003). According to common coding (James 1890; Prinz 1997), actions are cognitively represented in terms of their sensory consequences. Action observation may therefore lead to an internal activation of the action effects usually used to control one's own actions (motor simulation). Recently, evidence of motor simulation and action co-representation has accumulated when two individuals share one task (Sebanz et al. 2005). Classically, action co-representation has been investigated by using relatively simple spatial compatibility tasks (e.g., the Simon task) in an interactive context (Sebanz et al. 2003). In the basic Simon task, participants use a left and a right key to respond to non-spatial stimulus attributes, as for example, red and green dots that are presented either on the left or right side of a display. The Simon effect refers to the finding that participants' responses are slower when the spatial relationship between the stimulus side and the response side does not correspond than when it does correspond (De Jong et al. 1994). The Simon effect typically disappears when participants respond to only one of the colors in a Single go/nogo task (Liepelt et al. in pressb; Sebanz et al. 2003). When, however, another individual carries out the other part of the task, responding to the complementary color, the Simon effect reappears (Sebanz et al. 2003). When co-acting with another person, both participants seem to co-represent the other person's action (Sebanz et al. 2003, 2005) and activate the relative response codes in their action plans (Liepelt et al. in pressb).

Evidence of action co-representation has also been shown in other spatial compatibility tasks, as for example, in the social SNARC (spatial-numerical association of response codes) task (Atmaca et al. 2008). The SNARC effect (Dehaene 1992) describes the finding that participants respond faster to small numbers with a left key press than with a right key press, even when the magnitude of the number is taskirrelevant. This effect is explained by the assumption that the mental number line has a left to right (small to large) orientation that overlaps with the spatial response dimension. As in the Simon task, a social SNARC effect was present in a Joint go/nogo task, but not in a Single go/nogo task (Atmaca et al. 2008). These findings suggest that one's own and others' actions are represented in a functionally equivalent way when two individuals share one task.

However, little is known about the cognitive mechanisms underlying task sharing in more complex task situations. It is a completely open question whether two people sharing two tasks so that each person is in charge of only half the dual task show evidence of a social PRP (SPRP) effect. As we have described, the PRP effect has been assumed to be a central capacity limitation in information processing when one cognitive system has to deal with two tasks simultaneously. This raises the question of whether we can find evidence of bottleneck-like processing and shared task co-representations when a dual task is shared between two people. Answering this question seems fundamental, because it has implications for both, the understanding of capacity limitations and resource allocation of information processing and theories of action co-representation.

Therefore, we developed a social dual-task paradigm where two individuals share two tasks to compare dual-task performance within and across individuals. Using a social 
variant of the dual-task paradigm, the present study provides first evidence that a SPRP effect can be induced when two people share two tasks.

\section{Experiment 1}

To address the question of whether two people share a common action representation when performing a dual task in a social context, we compared the performance on PRP dual tasks in three different conditions (Single task, Joint task, and Dual task). The dual task was made of two number magnitude tasks. Participants had to indicate whether two numbers appearing one above the other were smaller or larger than five. Numbers were presented with a variable SOA. Participants responded to the upper number with lefthand button presses and to the lower number with righthand button presses. In the dual-task condition, a single person performed both responses. In the joint-task condition, the task was distributed between two people. Each individual responded to only one of the numbers. In the single-task condition, the identical number task was performed alone (Fig. 2). In all task conditions, both stimuli appeared on the screen.

For a social dual-task situation, common coding predicts that the second person acting on the second task monitors the first person's task that commences earlier in time. Therefore, one would predict an SPRP effect on RT 2 (Person 2) in the joint-task condition. This effect should be larger than in the single-task condition. In contrast theories that assume separate coding across persons predicts that two individuals have two separate brains and also two separate cognitive systems. Therefore, one would predict no SPRP effect on RT 2 (Person 2) in the joint-task condition. Joint-task performance should not differ from single-task performance.

Method

\section{Participants}

A group of 33 undergraduate students (age range: 20-35; mean age, 24.39 years; $\mathrm{SD}=3.79 ; 12$ female) participated in Experiment 1. Eleven were assigned to the dual-task group and eleven pairs performed the joint task and the single task. All were right-handed, had normal or corrected-tonormal vision, and were naive with regard to the hypotheses of the experiment. Participants were paid €7 for their participation. Participants gave a written informed consent to participate in the study, which was conducted in accordance with the ethical standards laid down in the 1975 Declaration of Helsinki.
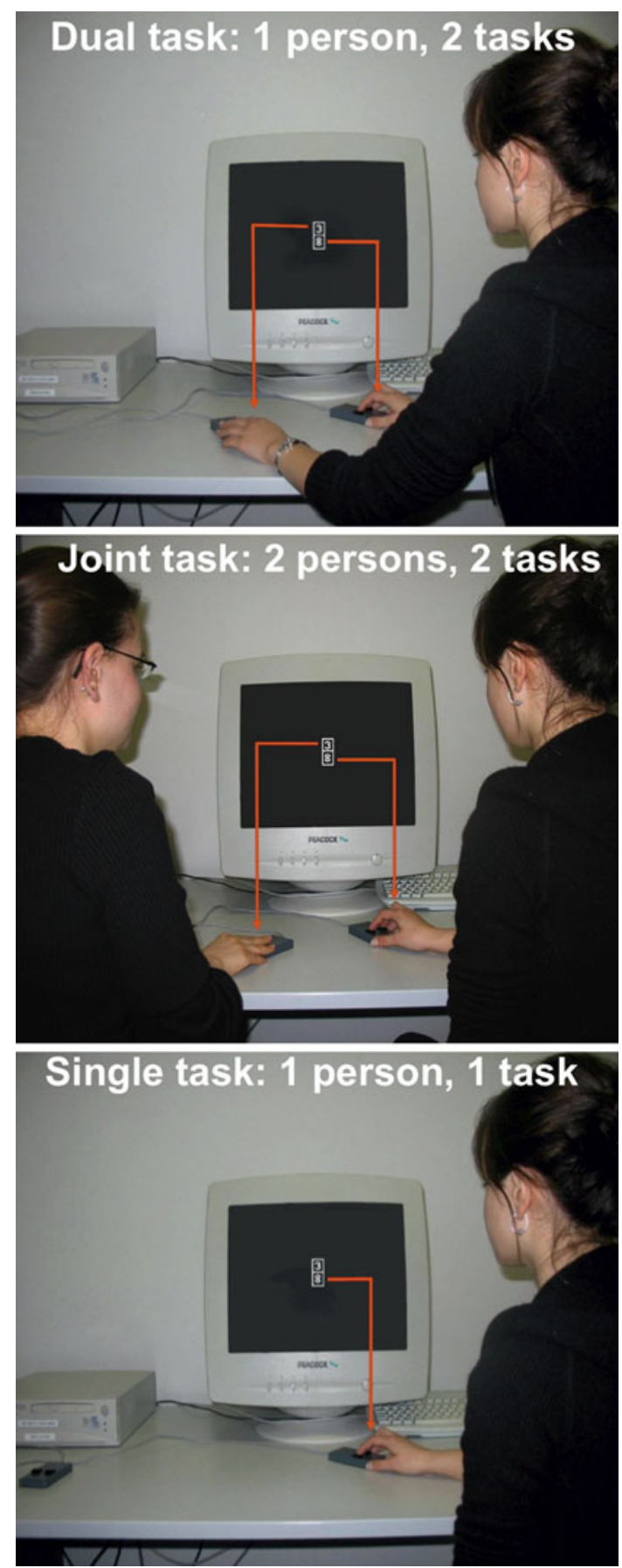

Fig. 2 Experimental set up used in Experiments 1 and 2. The upper panel shows the dual-task condition, where one person has to respond to two tasks using the index and middle finger of the left hand for the upper number stimulus (Task 1), and the right index and middle finger for the lower number stimulus (Task 2). The middle panel shows the joint-task condition, where two individuals share the two tasks. The person on the left has to respond with the index and middle finger of the right hand to the upper number stimulus (Task 1) and the person sitting on the right has to respond with the right index and middle finger to the lower number stimulus (Task 2). The lower panel shows the single-task condition, where one person has to respond to only one task. Here, the person sitting on the right has to respond with the index and middle finger of the right hand to the lower number stimulus (Task 2) 


\section{Apparatus and stimuli}

The experiment was conducted in a dimly lit, sound-attenuated room. The stimuli were displayed on a 17 -inch color monitor that was connected to a Pentium I PC. Stimuli were Arabic numerals (numbers: 1-4 and 6-9) in both tasks. Stimuli were presented with a variable SOA $(0,100,300$, $1,000 \mathrm{~ms}$ ) one above the other. Numbers were presented in white on a black background. Each digit was approximately $0.8 \mathrm{~cm}$ in width and $1.1 \mathrm{~cm}$ in height. The position of the character was indicated by a fixation stimulus, which consisted of a vertical box of $1.3 \mathrm{~cm}$ in width and $2.4 \mathrm{~cm}$ in height. The box was separated into two parts by a horizontal dash. It was presented in the center of the screen. At a viewing distance of $80 \mathrm{~cm}$, the box subtended $0.93^{\circ} \times 1.72^{\circ}$ of visual angle, with each number approximately $0.57^{\circ} \times 0.79^{\circ}$. Experiments were carried out using ERTS software (Beringer 2000). RTs and error rates were measured via button presses with a response box.

\section{Procedure and design}

Both tasks were number magnitude tasks. Participants had to indicate if the number(s) on the screen were smaller or larger than 5. In the dual-task condition, participants responded with the middle $(<5)$ and index finger $(>5)$ of the left hand to the upper number stimulus and with the index $(<5)$ and middle finger $(>5)$ of the right hand to the lower number stimulus. Participants sat on the left or right side in front of the monitor in the same position as in the joint and single tasks. The sitting position in the dual-task condition was counterbalanced across participants. In the joint task, participants were seated next to each other; in the dual-task and single-task conditions, an empty chair remained beside the participant. The sitting position between the joint and the single-task condition was kept constant: Participants who sat on the left side in the joint-task condition also sat on the left side in the single condition, and participants who sat right side in the joint-task condition also sat on the right side in the single-task condition. The logic of the sitting position was adapted to previous studies on the social Simon effect (Sebanz et al. 2003). Keeping the sitting position constant across single, joint, and standard-task conditions removes potentially confounds of the sitting position when comparing these conditions. In the single-task condition, one person had to respond to one task in isolation. In the joint-task condition, the task was distributed between two participants so that each individual had to respond to one stimulus. Participants performed the same task alone (single task) and together with a partner (joint task). In both the single-task and the joint-task conditions, participants responded with right-hand button presses with the index finger $(<5)$ and the middle finger $(>5)$. To control for perceptual differences across tasks, both number stimuli were presented on the screen in all three task conditions.

For both, dual-task and joint-task conditions, participants were instructed to give priority to Task 1 (Pashler 1994). We asked participants to concentrate and respond first to the upper number stimulus (Task $1 /$ Person 1) and then to the lower number stimulus (Task 2/Person 2). In all conditions, participants were encouraged to respond as quickly and accurately as possible.

Participants responded to numbers appearing in the upper or lower part of the fixation box. Each trial began with the presentation of the fixation box for 1,600 ms. Both numbers appeared for $200 \mathrm{~ms}$ (SOA $0 \mathrm{~ms}$ ). For the 100, 300 , and $1,000 \mathrm{~ms} \mathrm{SOA}$ conditions, the appearance of the second number stimulus was delayed by the respective interval. Responses had to be given within $1,800 \mathrm{~ms}$. If the response was correct, the screen remained blank. If no response was given within $1,800 \mathrm{~ms}$, the feedback "zu langsam" (too slow) was shown. In the case of an incorrect response, error feedback "Fehler" (error) was provided. All feedbacks (blank, too slow, or error) were displayed for $300 \mathrm{~ms}$. After the feedback, a constant inter-trial interval was given for $700 \mathrm{~ms}$. As described, 22 participants performed the single-task and joint-task conditions. Eleven of them performed 256 trials in Task 1, and eleven performed 256 trials in Task 2. In the dual-task condition, we had eleven participants performing 256 dual-task trials, so that they also had 256 Task 1 and 256 Task 2 stimulus contacts. Each large 256 trial block was split in four short blocks, each containing 64 trials intermitted by short breaks. We aimed to keep the trial contact per task and person constant across all task conditions. Participants started with the single-task condition, followed by the joint-task condition. The dual-task condition was performed with a separate group of participants.

\section{Results}

Errors (Task 1: $3.8 \%$, Task 2: $4.6 \%$ ) were excluded from further reaction time analyses. RT 1 and RT 2 were analyzed separately using a two-way analysis of variance (ANOVA) including the factors Type of Task (single task, joint task) and SOA $(0,100,300,1,000 \mathrm{~ms})$, both factors as within-subject variables. Additionally, we performed an ANOVA including Type of Task (joint task, dual task) and SOA $(0,100,300,1,000 \mathrm{~ms})$, with the former as a betweensubject variable and the latter as a within-subject variable.

\section{Reaction time analysis Task 2}

We found a significant effect of Type of Task, $F(1,10)=29.17, P<.001, \eta^{2}=.75$, with slower RTs in the joint-task condition $(612 \mathrm{~ms})$ as compared to the 
Fig. 3 Effects of stimulus onset asynchrony (SOA) on reaction times (RTs) under dual-task instructions giving priority to Task 1 for the dual-task (straight lines), joint-task (dotted lines) and single-task (dashed lines) conditions of Experiment 1. The left panel shows RTs for Task 2, and the right panel shows RTs for Task 1

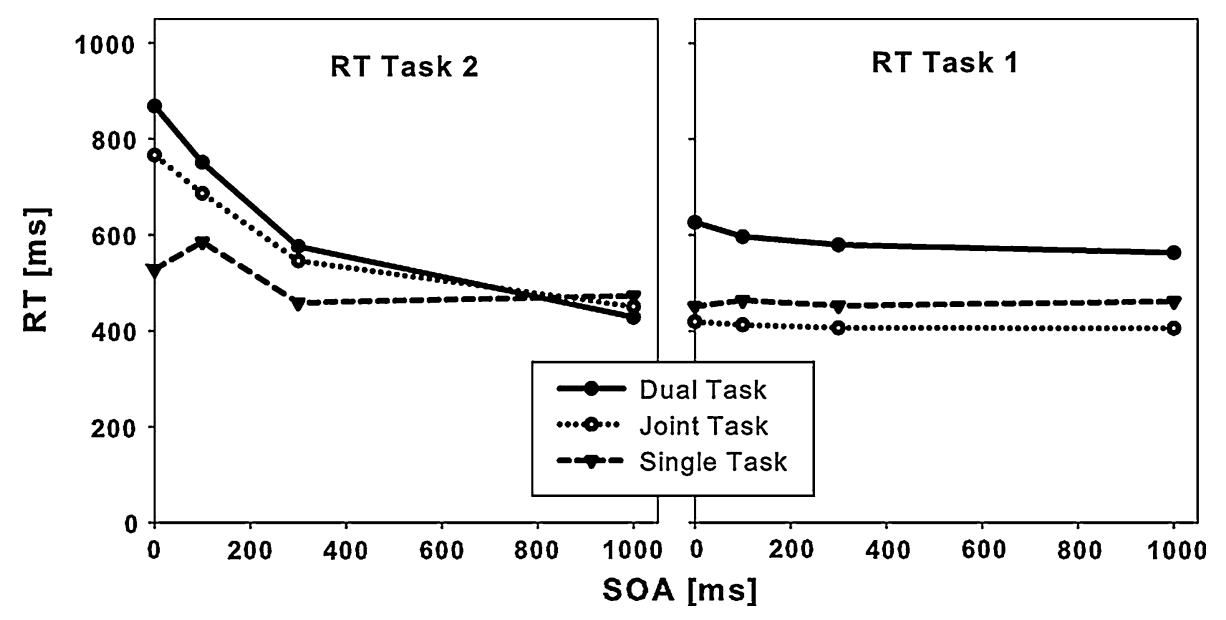

single-task condition $(511 \mathrm{~ms})$. The RTs increased with decreasing SOAs, as indicated by a significant effect of SOA, $F(3,30)=240.94, P<.001, \eta^{2}=.96$. This effect was significantly larger for the joint as compared to the singletask condition, as indicated by a significant interaction of Type of Task $\times$ SOA, $F(3,30)=60.10, P<.001, \eta^{2}=.86$. The difference was largest for the $0 \mathrm{~ms} \mathrm{SOA}$, amounting to $239 \mathrm{~ms}, P<.001$, followed by the $100 \mathrm{~ms}$ SOA, $101 \mathrm{~ms}$, $P<.05$ and the $300 \mathrm{~ms}$ SOA, $87 \mathrm{~ms}, P<.05$. For the $1,000 \mathrm{~ms} \mathrm{SOA}$, the difference was completely eliminated, $P>.45$ (Fig. 3, left panel). Comparing joint-task and dualtask conditions, we found no effect of Type of Task, $F(1,20)=2.79, P>.05, \eta^{2}=.12$, but numerically faster RTs in the joint-task condition (612 ms) as compared to the dual-task condition $(656 \mathrm{~ms})$. Again, the RTs increased with decreasing SOAs, $F(3,60)=411.52, \quad P<.001$, $\eta^{2}=.95$. This effect was larger for the dual-task, as compared to the joint-task condition, as indicated by a significant interaction of Type of Task $\times \operatorname{SOA}, F(3,60)=10.18$, $P<.05, \eta^{2}=.34$. While we found no difference between the dual-task and the joint-task conditions in the $1,000 \mathrm{~ms}$ SOA condition, the difference in the $300 \mathrm{~ms}$ SOA amounted to $30 \mathrm{~ms}, P<.05$, to $65 \mathrm{~ms}$ in the $100 \mathrm{~ms}$ SOA condition, $P<.05$, and was largest but statistically less reliable in the $0 \mathrm{~ms}$ SOA condition $(102 \mathrm{~ms})$.

\section{Reaction time analysis Task 1}

Comparing the joint-task and single-task conditions, we found a significant effect of Type of Task, $F(1,10)=11.96$, $P<.05, \eta^{2}=.55$, with faster RTs in the joint task $(411 \mathrm{~ms})$ as compared to the single-task condition (457 ms). Neither the effect of $S O A, F(3,30)<1, \eta^{2}=.05$, nor the interaction of Type of Task $\times S O A$ reached significance, $F(3$, $30)=1.59, P>.05, \eta^{2}=.14$. The Type of Task effect was present in all SOA conditions, all $P \mathrm{~s}<.05$ (Fig. 3, right panel). Comparing the joint-task and the dual-task conditions, we found a significant effect of Type of Task, $F(1,20)=36.48, P<.001, \eta^{2}=.65$, with faster RTs in the joint-task (411 ms) as compared to the dual-task condition $(591 \mathrm{~ms})$. We also found an increase in RTs with decreasing SOAs, $F(3,60)=13.70, P<.001, \eta^{2}=.41$. This effect was larger for the dual-task condition as compared to the joint-task condition, as indicated by a significant interaction of Type of Task $\times$ SOA, $F(3,60)=5.33, P<.05, \eta^{2}=.21$. The SOA effect was largest for the $0 \mathrm{~ms}$ SOA, amounting to $207 \mathrm{~ms}, P<.05$. The $184 \mathrm{~ms}$ difference in the $100 \mathrm{~ms}$ SOA was less reliable than the $173 \mathrm{~ms}$ difference in the $300 \mathrm{~ms}$ SOA and the $158 \mathrm{~ms}$ in the $1,000 \mathrm{~ms} \mathrm{SOA}, P \mathrm{~s}<.05$.

\section{Error analysis Task 2}

We performed the same analyses used for RTs also for error rates (Table 1, left panel). For errors, the comparison of single-task and joint-task conditions showed a significant effect of $S O A, F(3,30)=4.59, P<.05, \eta^{2}=.32$, which was due to a slight error increase at shorter SOAs present in both, single-task and joint-task conditions. Neither the effects of Type of Task, nor the interaction of Type of Type of Task $\times S O A$ reached significance, all $P \mathrm{~s}>.05$. When comparing joint-task and dual-task conditions, we found a significant effect of Type of Task, $F(1,20)=10.68, P<.05$, $\eta^{2}=.35$, with less errors in the joint-task condition as compared to the dual-task condition. Errors increased with decreasing SOAs, $F(3,60)=5.07, P<.05, \eta^{2}=.20$. This effect was larger for the dual-task, as compared to the jointtask condition, as indicated by a significant interaction of Type of Task $\times$ SOA, F(3, 60) $=3.04, P<.05, \eta^{2}=.13$. While the larger error rates in the dual-task as compared to the joint-task conditions was reliable at the $300 \mathrm{~ms}$ and $1,000 \mathrm{~ms}$ SOAs $(P<.05)$, we found smaller and less reliable differences for the two short SOAs (both $P$ s $>.05$ ). 
Table 1 Mean percent error (PE) for Single-task, Joint-task, and Dual-task conditions for the four Stimulus Onset Asynchronies (SOA 0 ms, SOA $100 \mathrm{~ms}$, SOA $300 \mathrm{~ms}$, and SOA 1,000 ms) separately for Task 2 (left panel) and Task 1 (right panel) of Experiment 1

\begin{tabular}{|c|c|c|c|c|c|c|}
\hline & \multicolumn{3}{|l|}{ Task 2} & \multicolumn{3}{|l|}{ Task 1} \\
\hline & Single task & Joint task & Dual task & Single task & Joint task & Dual task \\
\hline SOA 0 & 1.7 & 3.7 & 7.1 & 4.0 & 4.8 & 2.8 \\
\hline SOA 100 & 4.8 & 2.8 & 7.7 & 4.3 & 4.8 & 2.0 \\
\hline SOA 300 & 5.3 & 2.6 & 11.4 & 4.0 & 4.5 & 1.7 \\
\hline SOA 1,000 & 1.4 & 1.1 & 5.1 & 1.7 & 6.3 & 4.0 \\
\hline
\end{tabular}

\section{Error analysis Task 1}

When comparing the joint-task and single-task conditions (Table 1, right panel), we only found a significant effect of Type of Task, $F(1,10)=7.19, P<.05, \eta^{2}=.42$, with higher error rates in the joint task as compared to the single-task condition. Neither the effect of $S O A, F(3,30)<1, \eta^{2}=.02$, nor the interaction of Type of Task $\times$ SOA reached significance, $F(3,30)=1.53, P>.05, \eta^{2}=.13$. Comparing the joint-task and the dual-task conditions, we only found a significant effect of Type of Task, $F(1,20)=5.76, P<.05$, $\eta^{2}=.22$, with higher error rates in the joint-task as compared to the dual-task condition. Neither the effects of $S O A$, $F(3,60)=1.52, P>.05, \eta^{2}=.07$, nor the interaction of Type of Task $\times$ SOA, $F(3,30)<1, \eta^{2}=.004$ reached the level of significance.

\section{Discussion}

Under dual-task instructions giving priority to Task 1, we found the typical RT 2 increase, with decreasing SOA in the dual-task condition (PRP effect) as well as in the jointtask condition, mimicking the typical PRP pattern (SPRP effect). In line with the idea of common coding, the RT 2 increase in the joint-task condition was larger than in the single-task condition, providing evidence of an SPRP effect. This effect was, however, smaller than the PRP effect in the dual-task condition. We found a small increase in RT 2 with decreasing SOA also in the single-task condition when two number stimuli were presented consecutively. The mere perception of two stimuli falling in the same category seems to produce interference during target processing (Eriksen and Schultz 1979). For RT 1, we found evidence for a social facilitation effect with faster RTs in the jointtask condition as compared to the dual-task and the singletask conditions. However, the finding of increased error rates for the joint-task condition as compared to the single task condition in Task 1 indicated that the RT 1 social facilitation effect represents no real facilitation but can be explained by a Speed-accuracy Tradeoff (SAT) on Task 1 error rates. Most importantly, the analysis of error rates showed that the finding of the SPRP effect on response times was not due to a SAT. In general, the error rate results paralleled those found for reaction times though the error rate findings were less reliable.

\section{Experiment 2}

In Experiment 2, we tested whether the SPRP effect is due to task-specific monitoring requirements for Person 2, as implemented by dual-task instructions giving priority to Task 1 . We used a similar design as in the previous experiment; however, we changed the task instructions. We used equal priority instructions (Meyer and Kieras 1997a) for both tasks and persons. If the SPRP effect found in Experiment 1 was due to task-specific monitoring requirements based on the dual-task instructions given, then the increase in RT 2 (Person 2) should now be of approximately the same size in joint and single-task conditions.

Method

\section{Participants}

A new group of 22 undergraduate students (age range: 20 28 , mean age 23.23 years; $\mathrm{SD}=2.02 ; 11$ female) participated in Experiment 2. Participants gave a written informed consent to participate in the study, which was conducted in accordance with the ethical standards laid down in the 1975 Declaration of Helsinki.

\section{Apparatus and stimuli}

Apparatus and stimuli were identical to the previous experiment.

\section{Procedure and design}

The procedure and design were identical to the previous experiment. In Experiment 2, the order of conditions (joint task and single task) was counter-balanced across pairs of participants whereas the dual task was always performed at the end within the same group of participants. All participants 
Fig. 4 Effects of stimulus onset asynchrony (SOA) on reaction times (RTs) under equal priority instructions for the dual-task (straight lines), joint-task (dotted lines) and single-task (dashed lines) conditions of Experiment 2. The left panel shows RTs for Task 2, and the right panel shows RTs for Task 1

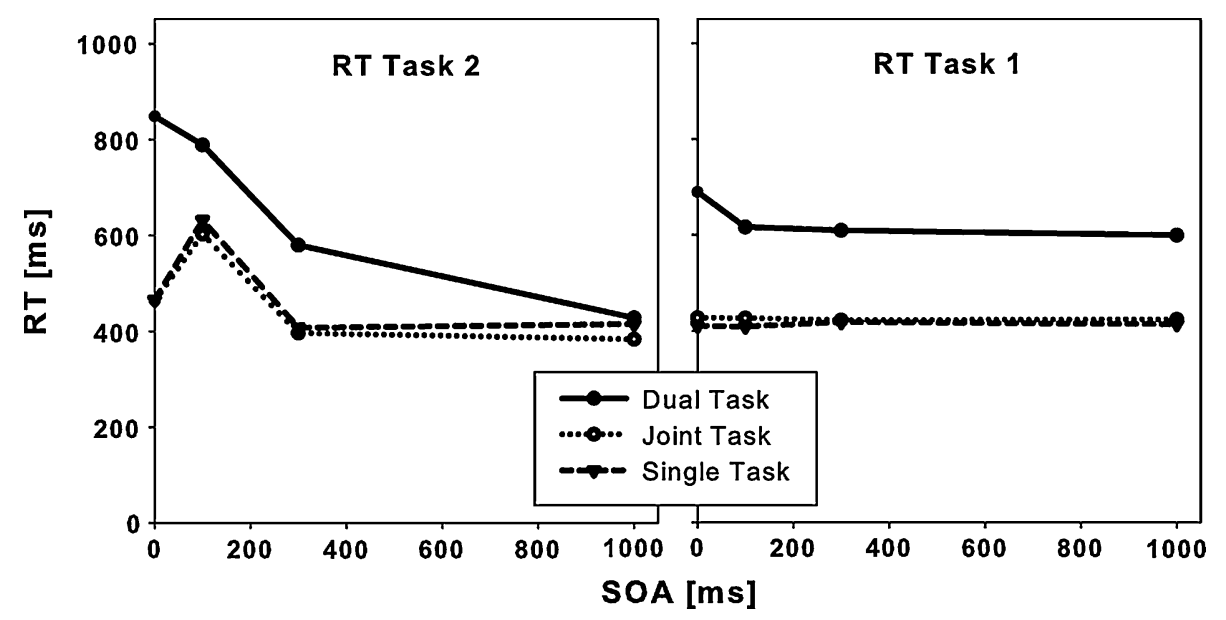

performed all three task conditions (single-task, joint-task, and dual-task conditions) completing 256 trials in both, Task 1 and Task 2. As in Experiment 1, each large 256 trial block was split in four short blocks, each containing 64 trials intermitted by short breaks. The sitting position was kept constant across all three task conditions (single task, joint task, and dual task). Participants remained on their sitting position during the entire experiment. Most importantly, we changed the task instructions for both the joint and dualtask conditions; Participants were instructed to give equal priority to both tasks (Meyer and Kieras 1997a; Schumacher et al. 2001). We asked participants to respond as quickly and accurately as possible in both tasks.

Results

The data were analyzed in the same way as in Experiment 1, with the factors Type of Task and SOA with two-way ANOVAs both factors as within-subject variables. In Experiment 2, the comparison of joint-task and dual-task conditions was now analyzed as a within-subjects factor since these conditions were given to the same group of participants. Errors (Task 1: 0.6\%, Task 2: $1.0 \%$ ) were excluded from further reaction time analyses.

\section{Reaction time analysis Task 2}

For the comparison of the joint-task condition with the single-task condition, we found no effect of Type of Task, $F(1,10)<1, \eta^{2}=.07$. The RTs increased with decreasing SOAs, as indicated by a significant effect of SOA, $F(3,30)=529.27, P<.001, \eta^{2}=.98$. We found a significant interaction of Type of Task $\times \operatorname{SOA}, F(3,30)=3.85$, $P<.05, \eta^{2}=.28$, which was based on a difference of $22 \mathrm{~ms}$ due to faster RTs in the joint-task condition as compared to the single-task condition in the $1,000 \mathrm{~ms}$ SOA condition. For all other SOA conditions, RTs did not differ between the joint-task and the single-task conditions (all $P \mathrm{~s}>.18$ ).
Comparing the joint- and dual-task conditions, we found a significant effect of Type of Task, $F(1,10)=83.18, P>.05$, $\eta^{2}=.89$, with faster RTs in the joint-task condition (461 ms) as compared to the dual-task condition (649 ms). RTs increased with decreasing SOAs, $F(3,30)=226.86$, $P<.001, \eta^{2}=.96$. The effect was larger for the dual-task condition as compared to the joint-task condition, as indicated by a significant interaction of Type of Task $\times$ SOA, $F(3,30)=81.36, P<.001, \eta^{2}=.89$ (Fig. 4, left panel). We found a significant difference between the dual-task condition and the joint-task condition for all SOA conditions (SOA1000: $28 \mathrm{~ms}$, SOA300: $173 \mathrm{~ms}$, SOA100: $180 \mathrm{~ms}$, and SOA0 ms: $371 \mathrm{~ms}$, all $P \mathrm{~s}<.05$ ).

\section{Reaction time analysis Task 1}

Comparing joint- and single-task conditions, we found no effect of Type of Task, $F(1,10)<1, \eta^{2}=.03$. Further, we found no effect of $\operatorname{SOA}, F(3,30)=1.71, P>.20, \eta^{2}=.15$. However, we found a significant interaction of Type of Task $\times \operatorname{SOA}, F(3,30)=3.84, P<.05, \eta^{2}=.28$, which was due to a small RT increase in the joint as compared to the single task in the short SOA conditions. This increase was, however, not reliable (SOA $0, P>.55$ and SOA 100, $P>.13$ ). Comparing the joint- and the dual-task conditions, we found a significant effect of Type of Task, $F(1,10)=93.73, P<.001, \eta^{2}=.90$, with faster RTs in the joint-task (425 ms) as compared to the dual-task condition (646 ms). We observed an increase in RTs with decreasing SOAs, $F(3,30)=15.16, P<.001, \eta^{2}=.60$. This effect was larger for the dual-task as compared to the joint-task condition, as indicated by a significant interaction of Type of Task $\times$ SOA $, F(3,30)=13.26, P<.001, \eta^{2}=.57$ (Fig. 4, right panel). We found a significantly stronger RT slowing effect with decreasing SOA in the dual-task condition as compared to the joint-task condition (SOA1000: $193 \mathrm{ms,}$ SOA300: $210 \mathrm{~ms}$, SOA100: $203 \mathrm{~ms}$, and SOA0 ms: $281 \mathrm{~ms}$, all $P \mathrm{~s}<.001)$. 
Table 2 Mean percent error (PE) for Single-task, Joint-task, and Dual-task conditions for the four Stimulus Onset Asynchronies (SOA 0 ms, SOA $100 \mathrm{~ms}$, SOA $300 \mathrm{~ms}$, and SOA 1,000 ms) separately for Task 2 (left panel) and Task 1 (right panel) of Experiment 2

\begin{tabular}{|c|c|c|c|c|c|c|}
\hline & \multicolumn{3}{|l|}{ Task 2} & \multicolumn{3}{|l|}{ Task 1} \\
\hline & Single task & Joint task & Dual task & Single task & Joint task & Dual task \\
\hline SOA 0 & 0.5 & 1.0 & 1.0 & 0.4 & 0.8 & 0.5 \\
\hline SOA 100 & 1.3 & 1.8 & 1.0 & 0.6 & 1.2 & 0.5 \\
\hline SOA 300 & 0.5 & 1.4 & 1.0 & 0.5 & 1.0 & 0.2 \\
\hline SOA 1,000 & 0.6 & 0.9 & 0.5 & 0.2 & 1.0 & 0.3 \\
\hline
\end{tabular}

\section{Error analysis Task 2}

The comparison of single-task and joint-task conditions showed a significant effect of $S O A, F(3,30)=8.17, P<.05$, $\eta^{2}=.45$, which was due to a slight error increase in the shorter SOAs in both, single-task and joint-task conditions (Table 2, left panel). The Type of Task effect, $F(1,10)=$ $10.11, P<.05, \eta^{2}=.50$, indicated higher error rates in the joint-task condition as compared to the single-task condition. The interaction of Type of Type of Task $\times$ SOA, did, however, not reach significance, $F(3,30)<1, \eta^{2}=.07$. The comparison of the joint-task and the dual-task condition showed a significant effect of Type of Task, $F(1,10)=3.21, P \leq .001$, $\eta^{2}=.67$, with slightly more errors in the joint-task condition as compared to the dual-task condition (Table 2, left panel). For these two conditions, we did not find a significant interaction of Type of Task $\times$ SOA, $F(3,30)=1.49, P>.05$, $\eta^{2}=.13$.

\section{Error analysis Task 1}

The comparison of joint-task and single-task conditions only showed a significant effect of Type of Task, $F(1,10)=7.86$, $P<.05, \eta^{2}=.44$, with higher error rates in the joint task as compared to the single-task condition (Table 2, right panel). Neither the effect of $S O A, F(3,30)=2.89, P>.05, \eta^{2}=.23$, nor the interaction of Type of Task $\times S O A$ reached significance, $F(3,30)<1, \eta^{2}=.03$. Comparing the joint-task and the dual-task conditions, we also found a significant effect of Type of Task, $F(1,10)=7.66, P<.05, \eta^{2}=.43$, with higher error rates in the joint-task as compared to the dual-task condition (Table 2, right panel). Neither the effects of SOA, $F(3,30)=1.01, P>.05, \eta^{2}=.09$, nor the interaction of Type of Task $\times$ SOA, $F(3,30)<1, \eta^{2}=.07$ reached the level of significance.

\section{Discussion}

Using equal priority instructions, we found a small RT 2 increase with decreasing SOAs in the joint-task condition. This RT 2 increase did not differ from the increase found in the single-task condition. These two effects were, however, smaller than the RT 2 increase found in the dual-task condition. The direct comparison to the dual-task condition has, however, to be taken with care, because the dual-task condition was manipulated within the same group of participants in Experiment 2, which may have effects of power on the analyses.

Despite the use of equal priority instructions, we found evidence of a standard PRP effect in the dual-task condition. This result pattern confirms that the SPRP effect previously found in the joint-task condition is due to taskspecific monitoring requirements. In Experiment 2, we found no evidence of social facilitation for RT 1.

An additional interesting finding we observed for Task 2 in the joint-task condition and the single-task condition of Experiment 2 (as well as in the single-task condition of Experiment 1) was a RT 2 increase from the $300 \mathrm{~ms}$ SOA to the $100 \mathrm{~ms}$ SOA and then a drop at the zero ms SOA. One possible explanation for this finding is related to the notion that the mere perception of two stimuli falling in the same category leads to interference during target processing (Eriksen and Schultz 1979) when both number stimuli were presented consecutively. When Person 2 prepares a response to the lower stimulus and the upper stimulus appears slightly earlier in time as, in the $100 \mathrm{~ms}$ and the $300 \mathrm{~ms}$ SOA, target interference cannot be avoided. However, when both stimuli appear at the same time (zero ms SOA) Person 2 may be able to directly focus attention to Stimulus 2 (the lower number stimulus) reducing possible interference from Stimulus 1 (the upper number stimulus).

The error analyses showed that the finding of a similar response times increase with decreasing SOA in single-task and joint-task conditions cannot be attributed to a SAT. However, for both, Task 2 and Task 1, we found significantly higher error rates for the joint-task condition as compared to the single-task condition and also when compared to the dual-task condition. This error effect was, however, unspecific with respect to the SOA. These findings may indicate the presence of some kind of unspecific social interference effect also present in the joint-task condition under equal priority instructions, which is neither sensitive to the amount of temporal overlap between tasks nor was it task specific (Task 1, Task 2). 
The present findings are in line with the assumption that the SPRP effect found for the joint-task condition in Experiment 1, which was sensitive to the amount of temporal overlap between tasks, is due to task-specific monitoring.

\section{Conclusions}

Using a dual-task paradigm previous studies showed evidence for capacity limitations when one cognitive system has to process two tasks simultaneously. Most studies used the PRP effect, as a marker for structural (Pashler 1994) and/or strategic (Meyer and Kieras 1997a) bottleneck processing. In the present study, we examined if bottlenecklike processing can also be elicited when a dual task is shared between two individuals testing coordinated cognition and action in multi-person performance.

Using a social dual-task paradigm, the present study provides first evidence of a social PRP effect when two individuals share two tasks. This SPRP effect depends on the task demands given. While it was present under instructions giving priority to Task 1 (Pashler 1994), it was diminished under instructions that gave equal priority to both tasks (Meyer and Kieras 1997a, b; Schumacher et al. 2001). These findings are in line with the assumption that the given task-monitoring requirements produce the SPRP effect.

Common coding (James 1890; Prinz 1997) assumes a shared representational space for two people sharing a task (Sebanz et al. 2003). According to common coding, the SPRP effect may be explained by the assumption that Person 2 is internally simulating specific aspects of the task performed by Person 1 that commences earlier in time. One way to explain the present finding of a SPRP effect is the assumption that the performer of Task 2 may have internally monitored or supervised the action or the corresponding action effects produced by the performer of Task 1 . The finding of a diminished SPRP effect under equal priority instructions suggests that common coding for self and other does not occur automatically when two complex tasks are shared. This seems to be different to task sharing situations where two persons share one task, as in the social Simon paradigm where co-representation seems to be a more automatic process (Sebanz et al. 2003). One potential reason for this difference may be that the social PRP effect confronts participants with two tasks that, at least in principal, may be conducted separately.

Crucial for the question of whether a person monitors another person's task, in our social dual-task paradigm, seem to be the demands of the task. In the present study, task demands were manipulated via task instructions, changing the task requirements and thus eliciting social corepresentation. But which requirements are most central for
Task 1 monitoring producing the SPRP? For us, the most plausible explanation is that the Task 1 priority instruction used in Experiment 1 may have prompted participants to monitor the stimulus and/or the response of Person 1, which may lead to the SPRP. What seems fascinating here is not that participants are able to adopt their performance to specific monitoring requirements per se, but that task monitoring across two individuals is able to produce a response time distribution for Person 2 (SPRP) that mimics the typical PRP pattern.

The finding of a general error rate increase in the jointtask condition as compared to both, single-task and dualtask conditions found in Experiment 2 (and also for Task 1 in Experiment 1) may indicate that even under equal priority instructions a social dual task may additionally induce some kind of unspecific interference for both tasks and persons, which was, however, not sensitive to the amount of temporal overlap between both tasks. Even under social dual-task conditions, actions of another person may have an impact on one's own actions, even when the task that has to be performed does not require taking the actions of the other person into account (Sebanz et al. 2003).

If more cognitive processes, such as response selection (Pashler 1994), are also subject to co-representation cannot be answered with the present study, but this will be an exciting question for future research on coordinated cognition and action in multi-person performance. Further, the present finding of the SPRP effect shows the strength of task instructions on shared task performance. Our conclusions are in line with recent findings showing that co-representation does not only rely on online feedback about the other person's actions but can be grounded in offline information (Vlainic et al. 2010) such as task instructions.

Common coding across minds, as defined here, has remarkable similarities to the strategic bottleneck account proposed for individual dual-task processing (Meyer and Kieras 1997a, b). According to the EPIC model, structural limitations are mainly related to physical constraints of the peripheral motor systems. For individual task performance, task monitoring may be understood in terms of strategic variations that are based on participant's attempts to satisfy task demands (Howes et al. 2009) and particular task instructions. Participants adopt specific processing strategies varying from cautious to daring task scheduling (Meyer and Kieras 1997a) to optimize task performance and fulfill task demands. Which strategies are adopted in a specific dual-task situation mainly depend on the task demands that are set. In the social dual-task condition, these demands may relate to Task 1 priority. According to the strategic bottleneck account, Person 2 may adopt a more cautious task-processing strategy in order to satisfy these demands. In line with the Task 1 priority instruction given in Experiment 1, it seems likely that cautious task process- 
ing may relate to the login point of central task processes (Meyer and Kieras 1997b) in Person 2 depending on response execution monitoring in Person 1 when multi-person coordination is required. The present findings of the SPRP effect under conditions of shared dual-task processing cannot be easily explained with the assumption of a structural capacity limitation made by the Response Selection Bottleneck model (Pashler 1994) without making further assumptions. When a structural bottleneck would drive the SPRP effect, the effect should have been much more independent from the task instructions given. As we found a diminished SPRP effect under equal priority instructions, our findings are more in line with the assumption of voluntary strategic bottlenecks as with involuntary structural bottlenecks for a social dual task situation. Further research is clearly needed to specify and further test the idea of task monitoring for the SPRP effect under multi-person coordination requirements.

If this is true, can the standard PRP effect (within one person) found in the dual-task condition of the present study also be explained by task monitoring? Under the assumption of similar resources underlying social and standard PRP effects, the answer would be yes-this may be the case. However, we found the standard PRP effect to be larger than the SPRP effect, and we also found a standard PRP effect in the dualtask condition under equal priority instructions. In the standard PRP eye movements may take additional time when moving from the top digit to the bottom digit, lengthening Task 2 response times especially at short SOAs. Furthermore, the two tasks we used required bi-manual responses, which may have produced additional motor-output interference in short SOAs when one individual has to perform two tasks at the same time. Just like the SPRP effect in the jointtask condition, the standard PRP may be attributable to chosen strategies of task scheduling revealed by task instructions and the types of tasks chosen (Liepelt et al. 2011; Meyer and Kieras 1997a, b; Schumacher et al. 2001).

Taken together, the present findings provide first evidence of a social PRP effect when two tasks are shared. The concept of action co-presentation may be extended from one task (Sebanz et al. 2005) to two tasks, at least under certain dual-task conditions.

Acknowledgments The authors wish to thank Patricia Grocke for help with data acquisition.

Conflict of interest The authors declare that they have no conflict of interest.

\section{References}

Atmaca S, Sebanz N, Prinz W, Knoblich G (2008) Action co-representation: the joint SNARC effect. Soc Neurosci 3:410-420
Beringer J (2000) Experimental runtime system. BeriSoft Cooperation, Frankfurt am Main, 1987-2000

Brass M, Bekkering H, Wohlschläger A, Prinz W (2000) Compatibility between observed and executed finger movements: comparing symbolic, spatial, and imitative cues. Brain Cogn 44:124-143

De Jong R, Liang CC, Lauber E (1994) Conditional and unconditional automaticity: a dual-process model of effects of spatial stimulusresponse correspondence. J Exp Psychol Human 20:731-750

Dehaene S (1992) Varieties of numerical abilities. Cognition 44:1-42

Eriksen CW, Schultz DW (1979) Information processing in visual search: a continuous flow conception and experimental results. Percept Psychophys 25:249-263

Howes A, Lewis RL, Vera AH (2009) Rational adaptation under task and processing constraints: implications for testing theories of cognition and action. Psychol Rev 116:717-751

James W (1890) The principles of psychology (vols 1-2). Holt, New York

Liepelt R, von Cramon DY, Brass M (2008) What is matched in direct matching? Intention attribution modulates motor priming. J Exp Psychol Human 34:578-591

Liepelt R, Fischer R, Frensch PA, Schubert T (2011) Practice-related reduction of dual-task costs under conditions of a manual-pedal response combination. J Cogn Psychol 23:29-44

Liepelt R, Strobach T, Frensch P, Schubert T (in pressa) Improved inter-task coordination skills after extensive dual-task practice. Q J Exp Psychol

Liepelt R, Wenke D, Fischer R, Prinz W (in pressb) Trial-to-trial sequential dependencies in a social and non-social Simon task. Psychol Res

Meyer DE, Kieras DE (1997a) A computational theory of executive cognitive processes and multiple-task performance: part 1. Basic mechanisms. Psychol Rev 104:3-65

Meyer DE, Kieras DE (1997b) A computational theory of executive cognitive processes and multiple-task performance: part 2. Accounts of psychological refractory-period phenomena. Psychol Rev 104:749-791

Meyer DE, Kieras DE (1999) Précis to a practical unified theory of cognition and action: some lessons from EPIC computational models of human multiple-task performance. In: Gropher D, Koriat A (eds) Attention and performance XVII. Cognitive regulation of performance: interaction of theory and application. MIT Press, Cambridge, pp 17-88

Pashler H (1984) Processing stages in overlapping tasks: evidence for a central bottleneck. J Exp Psychol Human 10:358-377

Pashler H (1994) Dual-task interference in simple tasks: data and theory. Psychol Bull 116:220-244

Pashler H, Johnston JC (1989) Chronometric evidence for central postponement in temporally overlapping tasks. Q J Exp Psychol 41A:19-45

Prinz W (1997) Perception and action planning. Eur J Cogn Psychol 9:129-154

Schumacher EH, Seymour TL, Glass J, Lauber EH, Kieras DE, Meyer DE (2001) Virtually perfect time-sharing in dual-task performance: uncorking the central cognitive bottleneck. Psychol Sci 121:101-108

Sebanz N, Knoblich G, Prinz W (2003) Representing others' actions: just like one's own? Cognition 8:B11-B21

Sebanz N, Knoblich G, Prinz W (2005) How two share a task: corepresenting stimulus-response mappings. J Exp Psychol Human 6:1234-1246

Telford CW (1931) The refractory phase of voluntary and associative responses. J Exp Psychol 14:1-36

Vlainic E, Liepelt R, Colzato LS, Prinz W, Hommel B (2010) The virtual co-actor: the Social Simon effect does not rely on online feedback from the other. Front Cogn 1:1-6

Welford AT (1952) The "psychological refractory period" and the timing of high speed performance. Br J Psychol 43:2-19 